\title{
A comparison between histopathology and findings on magnetic resonance imaging of subcutaneous lipomatous soft-tissue tumors
}

\author{
Johannes Toirkens • Arthur M. De Schepper • Filip Vanhoenacker • Pieter Van Dyck • \\ Jan Gielen • David Creytens • Kristien Wouters • Matthias Eiber • Klaus Wörtler • \\ Paul M. Parizel
}

Received: 6 December 2010 /Revised: 27 February 2011/Accepted: 10 May 2011 /Published online: 24 May 2011

(C) European Society of Radiology 2011

\begin{abstract}
Objective To retrospectively analyse the magnetic resonance (MR) findings of subcutaneous lipomatous softtissue tumours (SLSTT) in order to define a diagnostic and therapeutic strategy.

Methods The MR findings of 46 SLSTT were registered for the following data: area, location within the subcutaneous compartment, dimensions, shape and delineation, signal intensity (SI) on different pulse sequences, signal homogeneity, degree and pattern of contrast enhancement. The MR findings, together with patients' age and gender, were compared with histopathological findings.

Results Sixty-eight percent of the lipomas had a homogeneous T1-SI identical to the SI of subcutaneous fat. Heterogeneity in other lipomas was due to fat necrosis and small septa. Other lesions with homogeneous T1-SI identical to the SI of subcutaneous fat included lipoblastoma, lip-
\end{abstract}

J. Toirkens $(\bowtie) \cdot$ A. M. De Schepper $\cdot$ F. Vanhoenacker

P. Van Dyck $\cdot$ J. Gielen $\cdot$ P. M. Parizel

Department of Radiology, University Hospital Antwerp,

Wilrijkstraat 10,

2650 Edegem, Belgium

e-mail: mennotoirkens@hotmail.com

D. Creytens

Department of Pathology, University Hospital Antwerp,

Edegem, Belgium

K. Wouters

Department of Scientific Coordination of Biostatics,

University Hospital Antwerp and University of Antwerp,

Edegem, Belgium

M. Eiber $\cdot$ K. Wörtler

Department of Radiology, Technische Universität München,

Munich, Germany omatosis and lipoma-like hibernoma. None of the liposarcomas were homogeneous and isointense to SI of subcutaneous fat on T1-weighted imaging (WI). Lipoma variants and liposarcomas showed overlapping MR characteristics.

Conclusions SLSTT with homogeneous T1-SI identical to the SI of subcutaneous fat are benign. If a SLSTT has other MR characteristics, differentiation based on MRI cannot be made and biopsy is needed. We used this finding in defining a diagnostic and therapeutic strategy for subcutaneous soft tissue tumours that are hyperintense on T1-WI.

Keywords Magnetic resonance imaging - Lipoma . Liposarcoma $\cdot$ Subcutaneous fat $\cdot$ Histopathology

\section{Introduction}

Lipomatous tumours are extremely common, especially the benign types, which are frequently located in the subcutaneous compartment [1]. The subcutaneous compartment is composed of adipocytes, small nutrient vessels and thin septa, and lies between the dermis and the superficial fascia of the underlying muscle layer, periosteum or tendons. Clinical examination alone is often insufficient to identify the nature and exact origin of the subcutaneous mass, in which case imaging is necessary. Ultrasound is often used as a first imaging technique because of its availability, low cost and ability to easily visualise multiple coexisting masses at different locations at the same time. However, there is controversy in the literature about the efficacy of ultrasound in correctly diagnosing lipomas. Magnetic resonance imaging (MRI) is an excellent diagnostic tool for detecting lipomatous components. To our knowledge there has not been a study 
that has specifically focused on the MRI characteristics of subcutaneous lipomatous soft-tissue tumours (SLSTT). The objective of this study was to compare histopathology with the MR findings in a large number of benign as well as malignant SLSTT and to use these findings in defining a diagnostic and therapeutic strategy.

\section{Materials and methods}

Forty-six lipomatous tumours were selected from a MR database of 355 histopathologically proven subcutaneous soft-tissue tumours of the Belgian Soft Tissue Neoplasm Registry (BSTNR) and the Technische Universität München collected between 2001 and 2010. Our material contained 38 benign and eight malignant SLSTT. The benign tumours consisted of lipoma $(n=22)$, lipoblastoma $(n=1)$, lipomatosis $(n=2)$, pleomorphic lipoma $(n=6)$, angiolipoma $(n=4)$ and hibernoma $(n=3)$. The malignant tumours consisted of welldifferentiated liposarcoma $(n=3)$, myxoid liposarcoma $(n=4)$ and pleomorphic liposarcoma $(n=1)$.

Three experienced musculoskeletal radiologists analysed the examinations together, using a scoring form (Table 1) while being blinded to the histopathological diagnosis. Age, gender, lesion area, location in the subcutaneous compartment, dimensions, shape, definition and signal intensity (SI) in different MR sequences were recorded. The BSTNR proposed routine MR protocol consisted of axial T1weighted images (WI), axial T2-WI, axial fat-saturated (FS) T1-WI, coronal or sagittal FS T2-WI depending on lesion location, axial contrast-enhanced (CE) FS T1-WI (intravenous injection of gadolinium chelates), coronal or sagittal CE FS T1-WI and subtraction of axial pre- and post-CE FS T1-WI. Nevertheless, a large number of MR studies from referring centres were performed with less or incidentally other sequences [short tau inversion recovery (STIR) or proton density imaging]. Because of the retrospective nature of the study this could not be corrected.

MR findings, together with patient age and gender, were compared with the histopathological findings based on the 2002 World Health Organization's (WHO) committee for the classification of soft-tissue tumours (STT) consensus. The excised specimens were not available for review or mapping correlation with the images, but the pathological reports were reviewed and compared with the MR findings.

\section{Results}

Forty-six of the 355 STT in our database were SLSTT, resulting in the following percentages: $6.2 \%$ lipomas, $4.5 \%$ lipoma variants and $2.3 \%$ liposarcomas.

Lipomas $(n=22)$ were found in 14 women and eight men. The mean age was 55 years (range 36-78 years). Most lipomas were located in the scapular compartment and the neck (both $18 \%$ ). Size was highly variable. The largest lipoma had a diameter of $150 \mathrm{~mm}$. Most lipomas $(n=15)$ were oval and 19 of them were sharply delineated. The location in the subcutaneous compartment was variable, making contact with the fascia as the most frequent finding. Table 2 shows the scores of the MR characteristics of lipomas.

Fifteen lipomas had a homogeneous T1-SI identical to the SI of subcutaneous fat. If available SI on T2-WI was also homogeneous and identical to the SI of subcutaneous fat and the SI on FS T1-WI and T2-WI was homogeneously suppressed. Figure 1 shows a typical example of a usual lipoma.

Heterogeneity on T1-WI was seen in seven cases due to fat necrosis or multiple septa. Lipomas contained thin septa (thickness $<2 \mathrm{~mm}$ ) in nine cases. Although subtle/moderate enhancement of the fibrovascular septa or capsule was seen in five cases, 12 of the 17 usual lipomas did not enhance after contrast agent administration.

Three out of the 22 lipomas contained histopathologically proven fat necrosis. The fat necrosis in one case presented on MRI with T1-WI and T2-WI hypointense, peripheral, curvilinear strands that enhanced after injection of gadolinium contrast medium (Fig. 2). In a second case it presented as a nodular component at the periphery of the lesion. This component was hypointense on T1-WI and hyperintense on T2-WI and showed subtle enhancement. Histopathology showed non-specific surrounding inflammation with fibrosis and calcification. Two other lipomas
Table 1 MR scoring form

\begin{tabular}{|c|c|c|}
\hline & Demographics & Age, gender \\
\hline & Area & $\begin{array}{l}\text { Head and neck, thoracic cage, abdominal wall, paraspinal, } \\
\text { pelvis, shoulder, upper arm, fore-arm-hand, thigh, knee, } \\
\text { lower leg and foot }\end{array}$ \\
\hline & Location in subcutaneous compartment & Touching dermis, fascia, both or none \\
\hline & Dimensions & Anteroposterior, craniocaudal, laterolateral diameter \\
\hline & Shape & Round, oval, polylobular, stellar, other \\
\hline$S I$ signal intensity, $W I$ weighted & Definition & Sharp, non-sharp \\
\hline $\begin{array}{l}\text { image, } F S \text { fat-saturated, } C E \\
\text { contrast-enhanced }\end{array}$ & SI & T1-WI, T2-WI, FS T1-WI, FS T2-WI, CE FS T1-WI \\
\hline
\end{tabular}


Table 2 MR characteristics of 22 subcutaneous lipomas

\begin{tabular}{|c|c|c|c|c|c|c|c|c|c|}
\hline T1-SI & $n$ & FS T1-SI & $n$ & T2-SI & $n$ & FS T2-SI & $n$ & CE FS T1-SI & $n$ \\
\hline$<$ SI of muscle & 0 & $<$ SI of muscle & 10 & $<$ SI of fat & 0 & $<$ SI of water & 14 & None & 12 \\
\hline$=\mathrm{SI}$ of muscle & 0 & $=\mathrm{SI}$ of muscle & 0 & $=\mathrm{SI}$ of fat & 18 & $=$ SI of water & 1 & Subtle/ moderate & 5 \\
\hline$>$ SI of muscle & 2 & $>$ SI of muscle & 0 & $>$ SI of fat & 1 & & & Marked & 0 \\
\hline$=\mathrm{SI}$ of fat & 20 & Not available & 12 & Not available & 3 & Not available & 7 & Not available & 5 \\
\hline Homogeneous & 15 & Homogeneous & 6 & Homogeneous & 14 & Homogeneous & 11 & Homogeneous & 5 \\
\hline Heterogeneous & 7 & Heterogeneous & 4 & Heterogeneous & 5 & Heterogeneous & 4 & Heterogeneous & 0 \\
\hline
\end{tabular}

SI signal intensity

contained non-lipomatous components on MRI. These nonlipomatous components presented as rounded intralesional areas on T1-WI, consisting of a slightly hypointense centre surrounded by a peripheral hypointense rim, or as cloudlike T1-WI hypointense stranding. Based on these findings, we retrospectively considered these two cases to be lipomas with fat necrosis.

The only case of lipoblastoma was found in a 13-yearold boy. The lesion, located in the paraspinal compartment, presented as an ill-defined, hardly visible, polylobular lesion touching dermis and fascia, homogeneous and isointense to subcutaneous fat on T1-WI and FS T2-WI. Histopathologically, the lobulated architecture was indicative of a mature lipoblastoma.

Lipomatosis $(n=2)$ was found in two men, aged 45 and 50 years. Mean diameter was $88 \mathrm{~mm}$. Both lesions touched the dermis. One case of lipomatosis presented as an ill-defined lipomatous mass in the nuchal region containing multiple intralesional, hypointense, thick septa on T1-WI and T2-WI. The other case consisted of multiple confluent lipomas in the left scapular region with identical SI as subcutaneous fat on T1-WI, FS T1-WI and T2-WI. There was no enhancement after contrast medium injection on FS T1-WI.

Pleomorphic lipomas $(n=6)$ presented as heterogeneous masses in the neck $(67 \%)$ or shoulder $(33 \%)$ with fatty and non-fatty signal characteristics. Four pleomorphic lipomas were found in men and the mean age was 55 years. The mean diameter was $63 \mathrm{~mm}$ (range 32-100 mm). Most (four) lesions were oval and touched the dermis and fascia. The lesions were of heterogeneous signal on T1-WI, T2-WI and FS sequences. The masses enhanced in a subtle to moderate way after contrast medium injection (Fig. 3).

Angiolipomas $(n=4)$ were equally divided between sexes with a mean age of 43 years. Half of the lesions were found
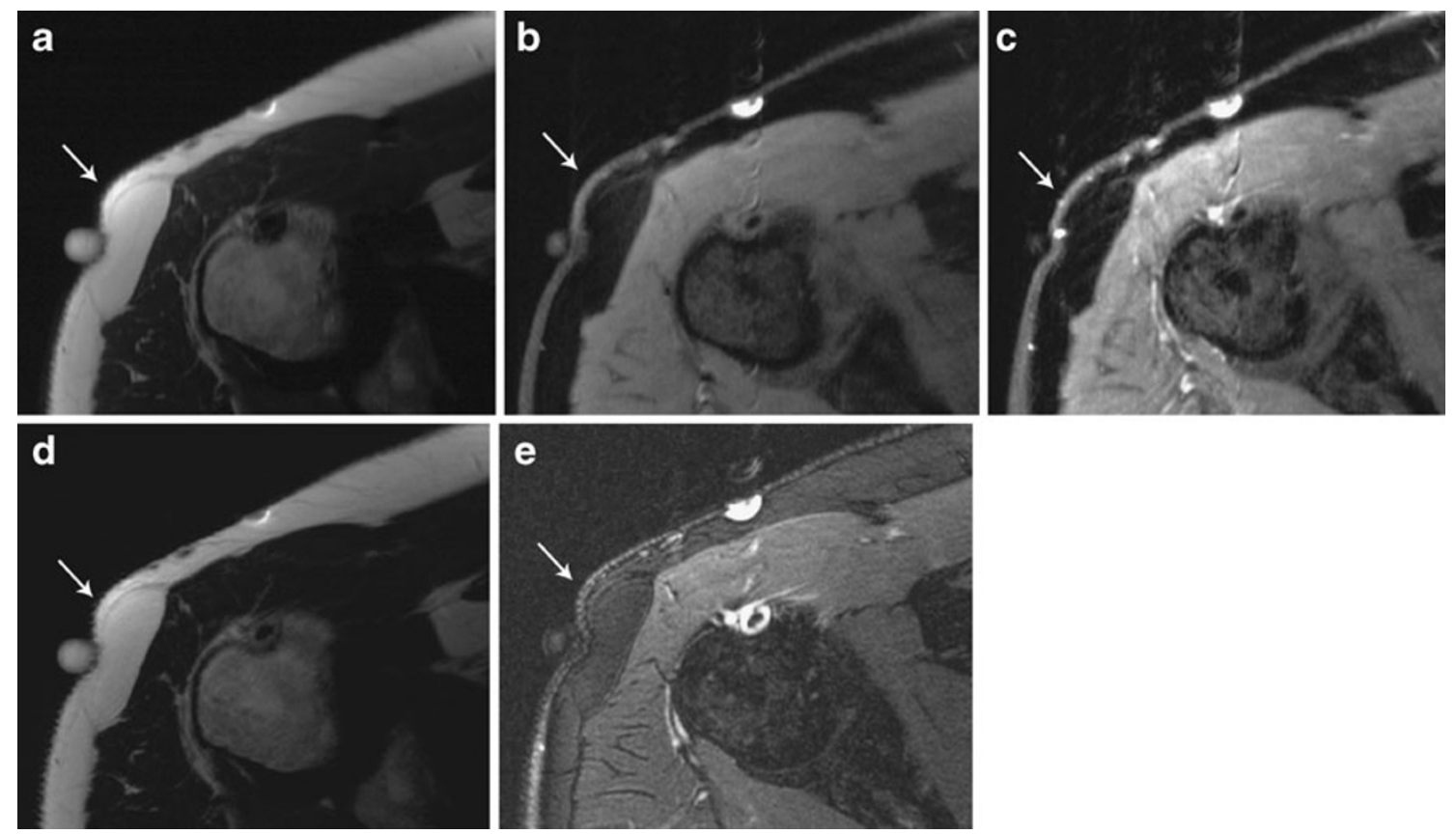

Fig. 1 Usual lipoma in the right scapular region of a 49-year-old man (arrow). a Axial T1-WI. b Axial FS T1-WI. c Axial CE FS T1-WI. d Axial T2-WI. e Axial FS T2-WI. The lesion has an SI identical to that of subcutaneous fat on all sequences. On FS sequences the SI of the lesion is homogeneously suppressed. No enhancement is seen 

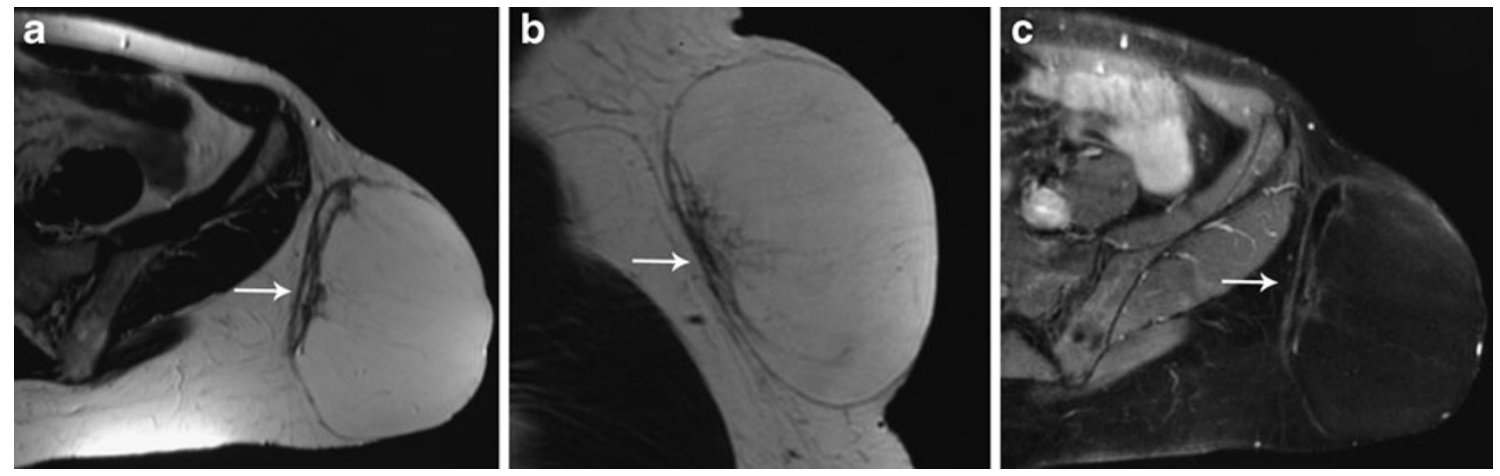

Fig. 2 Lipoma with fat necrosis and fibrous tissue in the left gluteal region of a 50-year-old woman (arrow). a Axial T2-WI. b Coronal T1WI. c Axial CE FS T1-WI. The tumour has fatty content with a

in the upper extremities. They presented as small, oval lipomatous masses (mean diameter $22 \mathrm{~mm}$ ). Most of them contained internal septa and small vessels that enhanced after contrast medium administration. The SI of the nonadipose components could not be or could only incompletely be suppressed. Because of these components the SI on T1-WI was heterogeneous in all cases. In one case, lesions were multiple.

Hibernoma $(n=3)$ was found in three women. The mean age was 44 years and mean diameter $79 \mathrm{~mm}$ (range 48$110 \mathrm{~mm}$ ). These sharply defined, oval/polylobular lesions were found in the neck, shoulder and thigh. All lesions were deeply located in the subcutaneous compartment and touched the fascia. The MR characteristics varied depending on the proportion of brown fat within the lesion. One hibernoma was isointense to fat on T1-WI and T2-WI, compatible with a lipoma-like hibernoma because it could not be distinguished from a usual lipoma based on these MR sequences. STIR images could identify the small brown lipomatous component with incomplete fat saturation (Fig. 4). The lesion showed a small component with subtle enhancement. Two hibernomas were of lower SI hypointense curvilinear area at the medial aspect of the lesion. There is subtle enhancement of this area

compared with fat on T1-WI and T2-WI, and were hyperintense on STIR images and enhanced markedly. Histopathologically, these lesions were classified as nonlipoma-like hibernomas and contained relatively more brown fat (Fig. 5).

Well-differentiated liposarcomas $(n=3)$ were found in three men aged between 19 and 62 years, in the shoulder, thigh and paraspinal region (Fig. 6). The mean diameter was $30 \mathrm{~mm}$ (range 19-41 mm). These oval/polylobular lesions had variable locations in the subcutaneous compartment. The MR appearances were also highly variable, with different SI on available pulse sequences. The only characteristic they had in common was heterogeneous SI on all pulse sequences.

Myxoid liposarcoma $(n=4)$ was found in four men, with a mean age of 41 years. All presented as sharply delineated, polylobular lesions (Fig. 7). Two lesions were located in the pelvic region, one in the thigh and one in the foot. The mean diameter was $70 \mathrm{~mm}$ (range $24-123 \mathrm{~mm}$ ). All lesions were deeply located in the subcutaneous compartment. The lesions were hypointense on T1-WI and hyperintense on T2-WI, corresponding to myxoid matrix on histopathology.
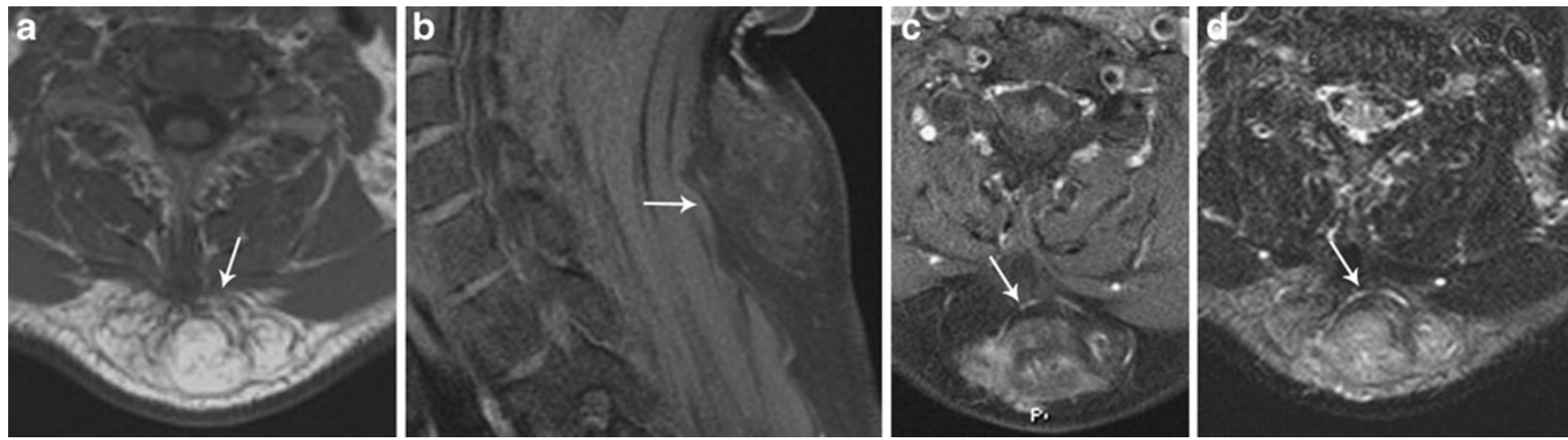

Fig. 3 Pleomorphic lipoma in the neck of a 61-year-old man (arrow). a Axial T1-WI. b Sagittal FS T1-WI. c Axial contrast-enhanced FS T1-WI. d Axial FS T2-WI. Heterogeneous mass with fatty and enhancing non-fatty components. Parts of the lesion remain hyperintense on T2-WI with fat suppression indicating the non-adipose components 
Fig. 4 Lipoma-like hibernoma in the anterior aspect of the left thigh in a 39-year-old woman (arrow). a Axial T1-WI. b Axial T2-WI. c Axial FS T1-WI. d Axial CE FS T1-WI. e Coronal STIR image. The mass is isointense to subcutaneous fat on T1-WI and T2-WI and shows subtle peripheral contrast enhancement. The lesion contains thin intralesional septa and has a peripheral capsule. STIR images show incomplete signal suppression
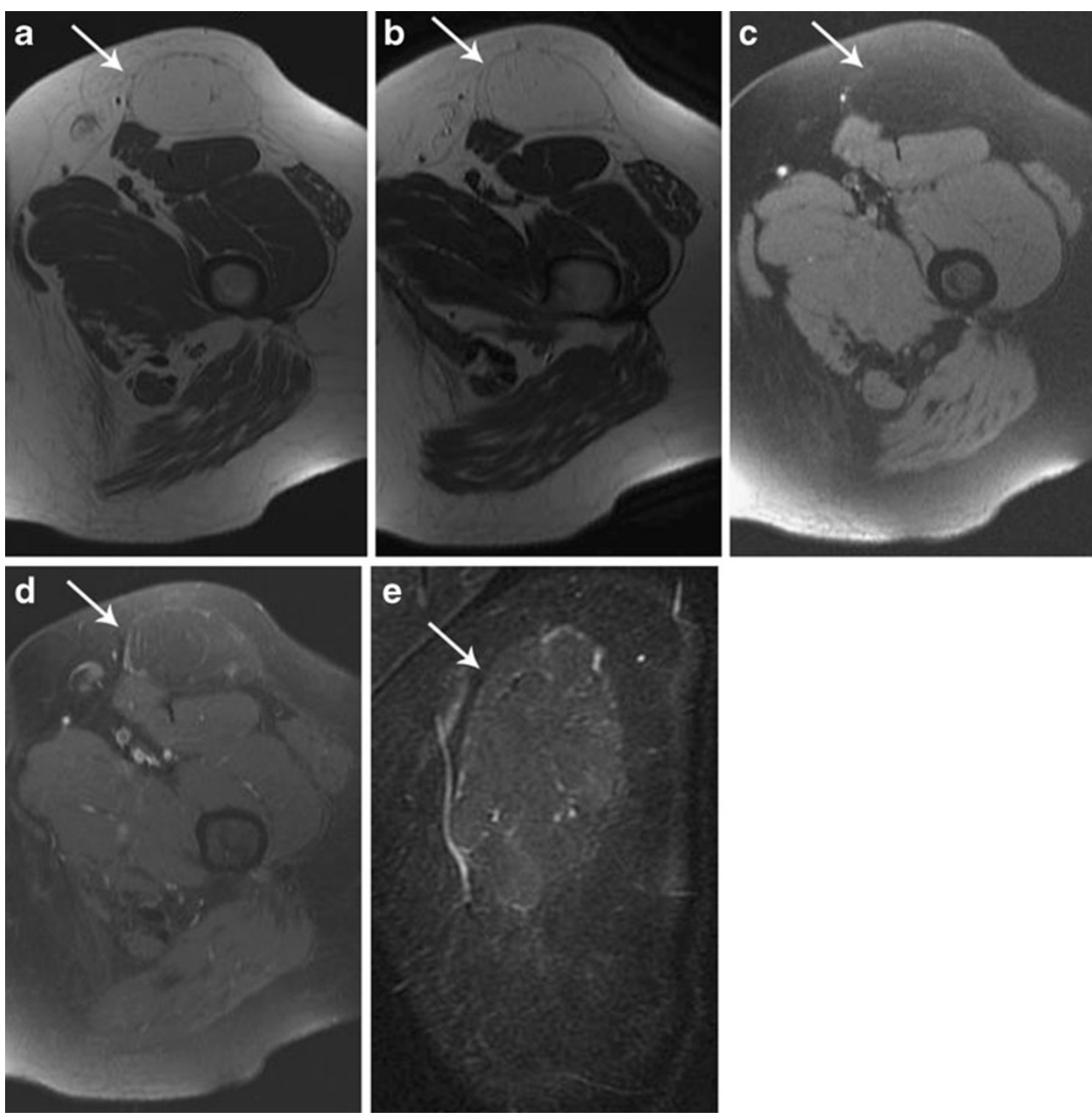

No lesion contained macroscopically visible fat on MRI. Three lesions enhanced markedly after contrast medium administration, two with a peripheral pattern and one with a central pattern.

Pleomorphic liposarcoma $(n=1)$ presented as a large, non-sharply delineated, polylobular mass in the left elbow in a 65-year-old man. The lesion had a diameter of $110 \mathrm{~mm}$ and touched the dermis and fascia. The SI of this tumour was heterogeneous on all pulse sequences (Fig. 8). We could not identify any macroscopically fatty component. Marked heterogeneous contrast enhancement was seen.

\section{Discussion}

Lipoma and lipomatous variants are the most frequent benign STT, with prevalence ranging between $16 \%$ and $50 \%$ [1-4]. Most of the benign lesions are located in the subcutaneous compartment of the superficial soft tissues [3,5]. Although liposarcoma is the second most frequent malignant STT (14.2\%) after myxofibrosarcoma, formerly named myxoid malignant fibrous histiocytoma [6], it is rather uncommon in the subcutaneous compartment [4]. Most liposarcomas are located in the deep parts of the extremities, especially in the thigh, or in the retroperitoneum. Differentiation is important because benign SLSTT can be treated conservatively or with marginal resection, while malignant SLSTT have to be resected with a wide margin. Although they rarely or never metastasize, even well-differentiated liposarcomas of the subcutaneous compartment, also called atypical lipomas [7, 8], need a wide resection margin to prevent recurrence and dedifferentiation [9].

The prevalence of various subtypes of SLSTT in our STT database is lower than those mentioned in the literature, but averaged over both benign and malignant soft tissue tumours and only valid for subcutaneous lesions. The low prevalence in our series is probably also due to selection bias, because many superficial lipomas are resected without pre-operative MR examination and even without histopathological confirmation. Furthermore, a number of small lipomas may be managed with clinical follow-up.

In accordance with literature data, lipomas were most frequently located in the head-neck region and trunk [2]. The location of lipoma variants and liposarcomas is more 
Fig. 5 Non-lipoma-like hibernoma in the right scapular region in a 63-year-old woman (arrow). a Sagittal T1-WI. b Axial T2-WI. c Sagittal STIR image. d Sagittal CE T1-WI. e Axial CE FS T1-WI. The mass is hypointense compared with subcutaneous fat on T1-WI and T2-WI, hyperintense on STIR images and shows marked diffuse contrast enhancement
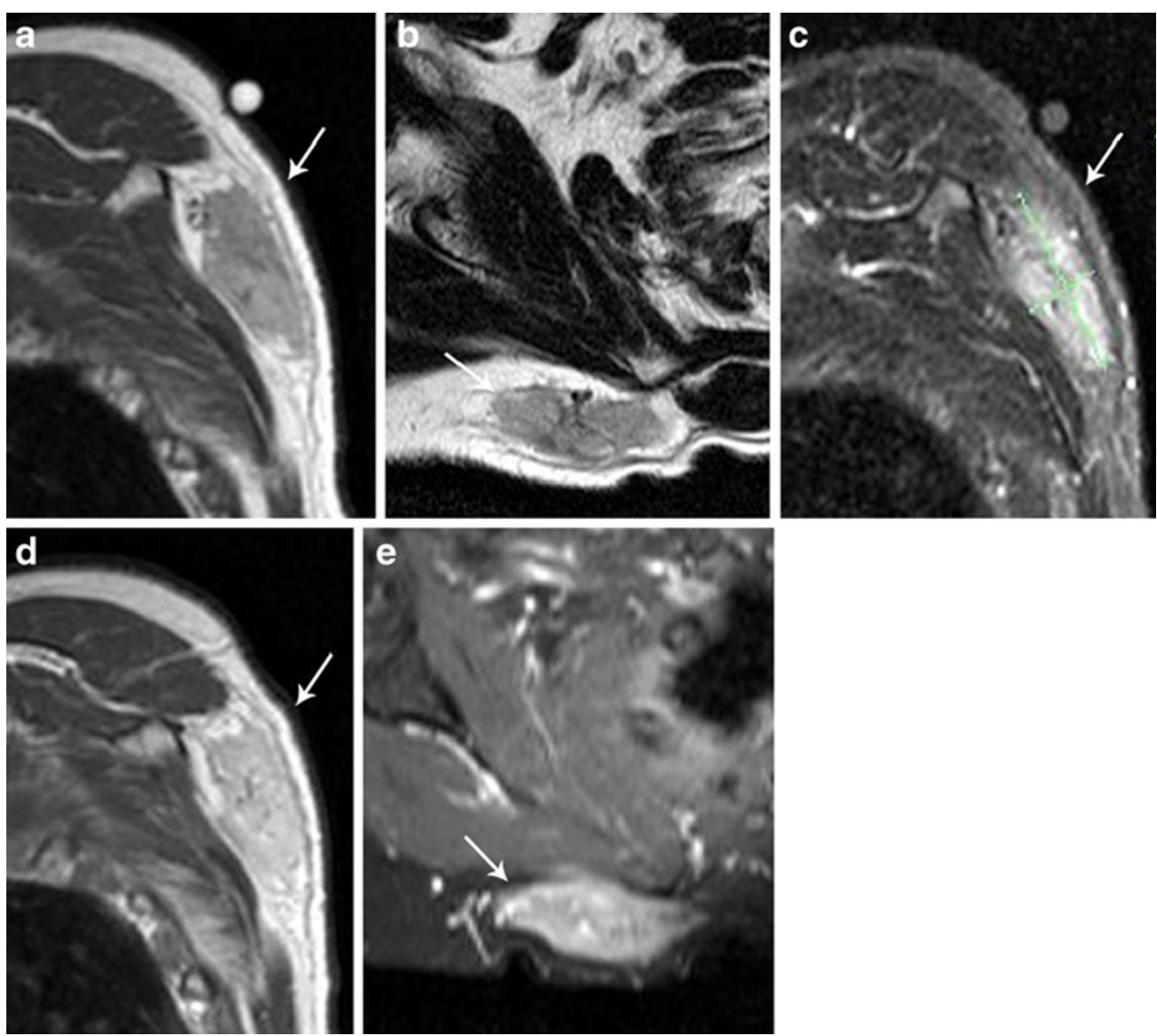

difficult to correlate with literature data because of the small number of cases in our series. Myxoid liposarcomas were found in the buttocks (two of four) and thigh (one of four), which are known as preferential locations [6]. Pleomorphic lipomas were found in the head-neck region and shoulder, which are sites of predilection [2, 10]. Moreover, pleomorphic lipoma/spindle cell lipoma (clinically and pathologically related variants) are most frequently seen in older ( $>60$ years) male patients. In our series, four out of six patients were male. For lipomas or other lipoma variants no definite sex predilection was noted or has been described.

Some authors described size as a significant differential parameter. Kransdorf et al. [11] found an odds ratio of 14.2 favouring a diagnosis of liposarcoma versus lipoma when a lipomatous lesion is larger than $10 \mathrm{~cm}$. In our series, the mean diameter of lipomas was $6.4 \mathrm{~cm}$. Surprisingly, lipomas were larger than the well-differentiated liposarcomas. Overall, size was not a useful parameter to differentiate benign from malignant SLSTT. Undersampling may be a reason for
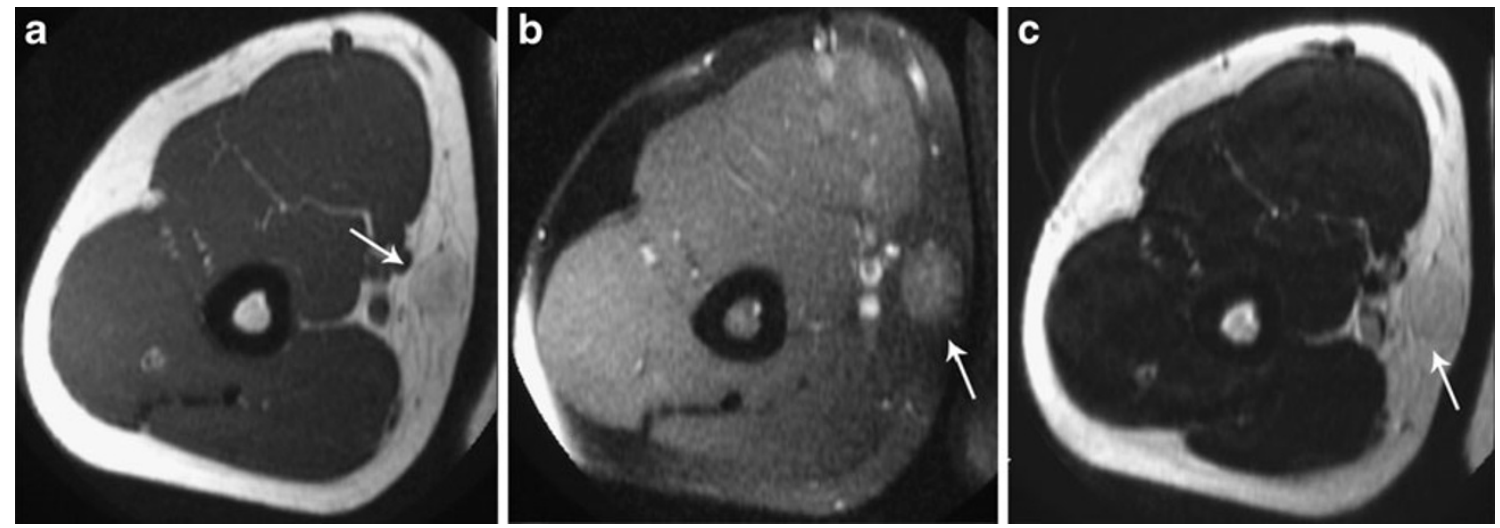

Fig. 6 Well-differentiated liposarcoma in the right upper arm of a 46year-old man (arrow). a Axial T1-WI. b Axial CE FS T1-WI. c Axial T2-WI. Subcutaneous oval-shaped mass with heterogeneous SI on T1-
WI and T2-WI. Parts of the tumour have lower T1 SI than the SI of subcutaneous fat and enhance with a nodular pattern 


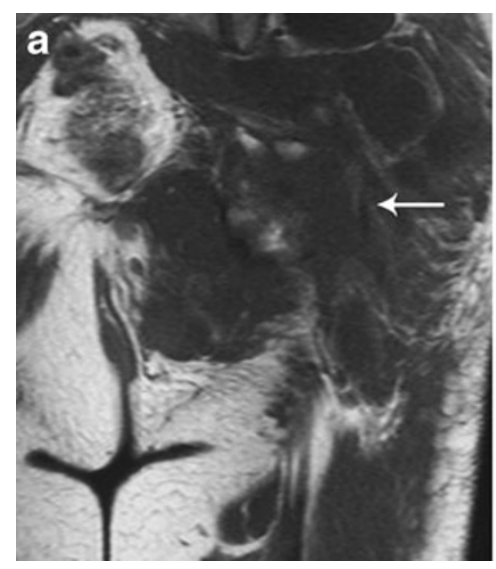

Fig. 7 Myxoid liposarcoma in the left buttock of a 47-year-old man (arrow). a Coronal T1-WI. b Axial FS T2-WI. c Sagittal FS T2-WI. Large polylobular lesion of low SI on T1-WI and high SI on T2-WI.
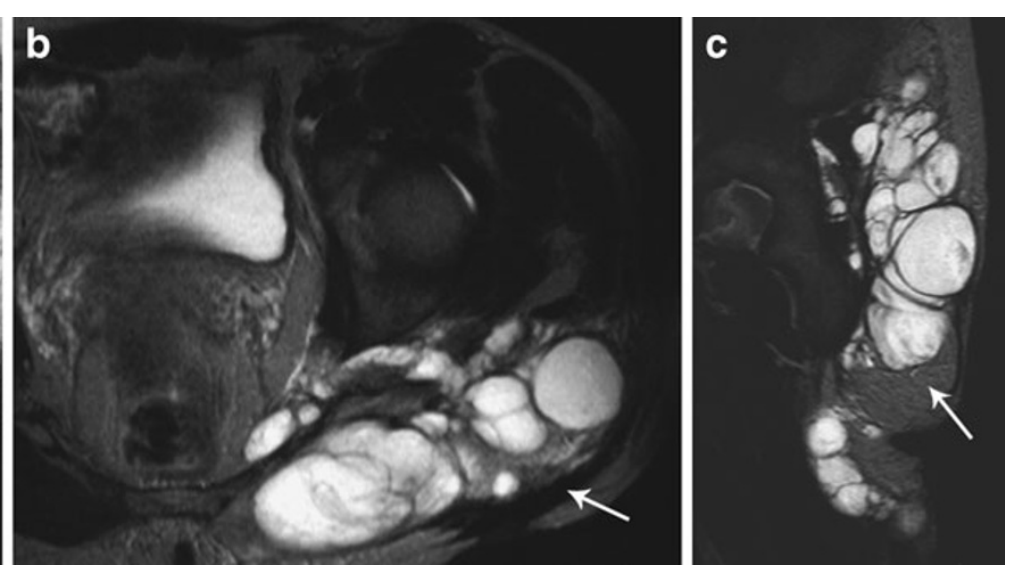

these equivocal results. However, the previously mentioned selection bias is probably also partly responsible.

The location in the subcutaneous compartment was variable in most lipoma and liposarcoma subgroups. Galant et al. [12] described malignant tumours of the subcutaneous compartment to have a higher tendency to develop a close relationship with the fascia than benign lesions. The only conclusion we can make is that the larger the lesion is, the more frequently it will touch the dermis and fascia. Galant et al. also stated that obtuse angles between superficial fascia and a subcutaneous mass or a lesion crossing the fascia would strongly suggest malignancy. Most of our subcutaneous liposarcomas (five of eight) did not cross the fascia nor did they have obtuse angles on MRI. However, none of the lipomas or lipoma variants had these features. The histopathological reports unfortunately did not describe the relationship of the tumours with the fascia.
Notice the presence of intralesional septa and intrapelvine extension. Macroscopically, no fatty components can be identified on different pulse sequences

Analysing the MR characteristics, we can distinguish three groups of SLSTT:

1. Usual lipomas. We found subcutaneous lipomas to have homogeneous T1-SI, identical to the SI of subcutaneous fat in 15 of the 22 cases $(68 \%)$. Heterogeneity was caused by fat necrosis in five cases and by multiple thin strands in two cases. These strands did not significantly enhance. Kransdorf et al. [11] already described slight heterogeneity and/or thin strands with mild to moderate enhancement in lipomas, reflecting the presence of fibrous tissue and blood vessels.

2. Lipomatous lesions containing non-adipose components. Our findings showed overlapping SIs for lipomas with fat necrosis, lipoma variants and liposarcomas, reflecting the variable composition and percentage of non-lipomatous components.
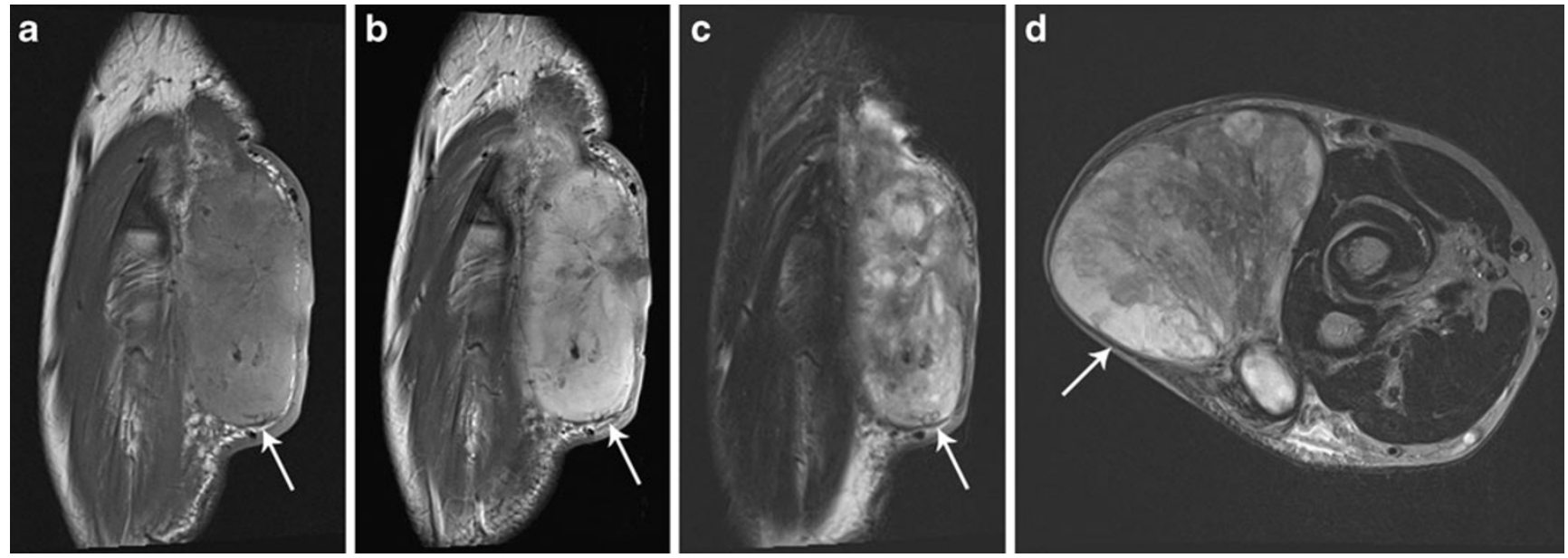

Fig. 8 Pleomorphic liposarcoma in the left elbow of a 65-year-old man (arrow). a Sagittal T1-WI. b Sagittal CE T1-WI. c Sagittal STIR image. d Axial T2-WI. The mass is heterogeneous on all pulse sequences. Macroscopically, no lipomatous component could be identified. Marked heterogeneous enhancement was seen 
3. Indeterminate lesions. SLSTT without gross lipomatous components, making identification of an SLSTT impossible. This was the case in eight SLSTT in our study (one pleomorphic lipoma, two hibernomas, four myxoid liposarcomas and one pleomorphic liposarcoma).

This leads us to the following diagnostic approach (Fig. 9):

- If a subcutaneous lesion has a homogeneous T1-SI identical to the SI of subcutaneous fat, the first differential diagnosis is a benign SLSTT. In our study, only usual lipoma, lipoblastoma, lipomatosis or lipomalike hibernomas had this homogeneous hyperintensity on T1-WI [13]. This may have important therapeutic consequences. Either a watchful waiting policy or a marginal lesion resection for aesthetic reasons may be considered. Theoretically, other subcutaneous lesions with high T1-SI should be excluded: lesions containing methaemoglobin, melanin or proteinaceous material [14]. The lipomatous origin can definitively be determined if the signal of the lesion is suppressed on FS T1WI [15]. Nevertheless, the probability of a nonlipomatous subcutaneous STT being homogeneously isointense with subcutaneous fat on T1-WI is very low in our opinion. An important consequence of this finding is that contrast medium administration is needless in these cases.

- If a subcutaneous fatty lesion contains non-adipose components, additional MR sequences are mandatory (T2-WI, fat suppression, contrast medium administration). In distinguishing lipoma from well-differentiated liposarcoma Galant et al. [16] advocated the use of FS T2-WI or STIR sequences. The presence of hyperintense septa or nodules on FS T2/STIR sequences would be more specific for well-differentiated liposarcoma than using the presence of thick septa or nodules on T1-WI as criteria of malignancy. In our study, we cannot confirm or reject this hypothesis due to an insufficient number of welldifferentiated liposarcomas. However, even with an extended MR protocol, tissue characterisation of these lesions is often not possible. Referral to an oncological centre is important to define the best therapeutic strategy. A biopsy will be often necessary and should be performed under computed tomography (or MR) guidance in order to obtain specimens of both components.

- This strategy should be the same for lesions without macroscopically visible lipomatous components.

Apart from this approach, and although statistical analysis was not feasible because of an insufficient number of different lipoma variants and liposarcomas, we noted some interesting trends:

- A lipoma containing a rounded or geographically shaped component with a T1-hypointense rim or peripheral hypointense strands is suggestive of intralesional fat necrosis. Chan et al. [17] reported similar cases with MR appearance of fat necrosis within lipomas.

- A rounded, heterogeneous lesion in the neck of a middle-aged man is highly suggestive of pleomorphic lipoma [10]. Bancroft et al. [18] reported similar MR characteristics in nine patients.

Our study has several weaknesses. It is a retrospective study in an existing database of histopathologically proven subcutaneous STT, making prospective conclusions based on MR findings only theoretical. We had limited numbers of each SLSTT subtype, especially from the liposarcomas. This renders statistical analyses impossible. Most of the studies in our database were performed in a referring institution using variable MR protocols, although a routine MR protocol was advised.

In conclusion, SLSTT with homogeneous T1-SI identical to the SI of subcutaneous fat are benign. Use of a simplified

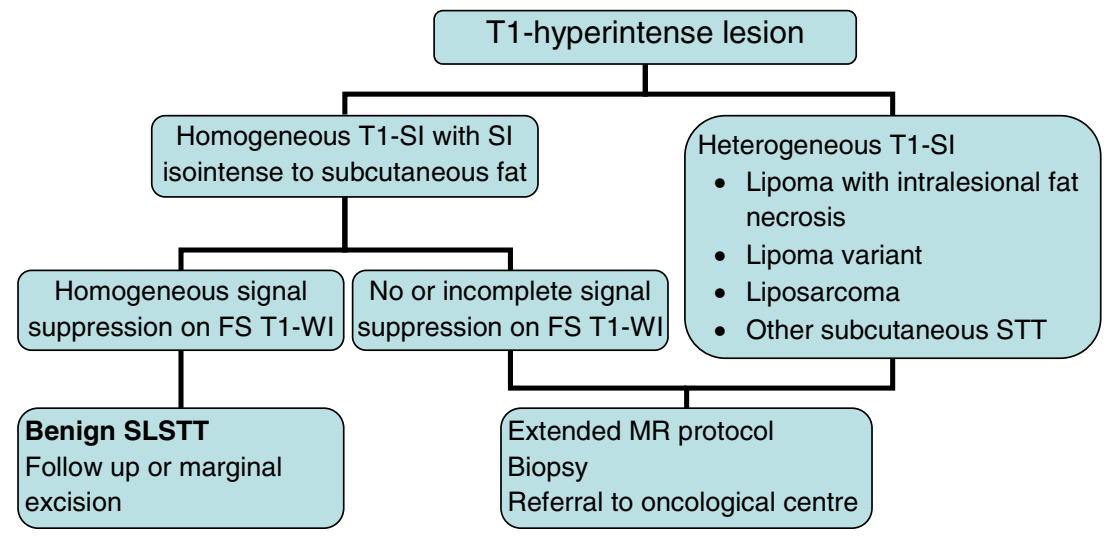

Fig. 9 Diagnostic and therapeutic algorithm for T1-hyperintense lesions. SI signal intensity, FS fat-saturated, STT soft-tissue tumour, SLSTT subcutaneous lipomatous soft-tissue tumour 
MR protocol consisting of T1-WI allows the benign nature of the SLSTT to be identified. If a SLSTT has other MR characteristics, differentiation based on MRI cannot be made, making biopsy and referral to an oncological centre necessary.

\section{References}

1. Myhrejensen O (1981) A consecutive 7-year series of 1331 benign soft-tissue tumors - clinicopathologic data-comparison with sarcomas. Acta Orthop Scand 52(3):287-293

2. Kransdorf MJ (1995) Benign soft-tissue tumors in a large referral population: distribution of specific diagnoses by age, sex, and location. AJR Am J Roentgenol 164(2):395-402

3. Blacksin MF, Ha D-H, Hameed M, Aisner S (2006) Superficial soft-tissue masses of the extremities. Radiographics 26:12891304. doi:10.1148/rg.265055729

4. Beaman DB, Kransdorf MJ, Tricia RA, Murphey MD, Arcara LK, Keeling H (2007) Superficial soft-tissue masses: analysis, diagnosis, and differential considerations. Radiographics 27:509-523. doi:10.1148/rg.272065082

5. Vanhoenacker FM, Marques MC, Garcia H (2006) Lipomatous tumors. In: De Schepper AM, Vanhoenacker F, Gielen J, Parizel PM (eds) Imaging of soft-tissue tumors. Springer, Berlin, pp 227261

6. Kransdorf MJ (1995) Malignant soft-tissue tumors in a large referral population: distribution of diagnoses by age, sex, and location. AJR Am J Roentgenol 164(1):129-134

7. Gaskin CM, Helms CA (2004) Lipomas, lipoma variants, and well-differentiated liposarcomas (atypical lipomas): results of MRI evaluations of 126 consecutive fatty masses. AJR Am J Roentgenol 182(3):733-739

8. Evans HL, Soule EH, Winkelmann RK (1979) Atypical lipoma, atypical intramuscular lipoma, and well-differentiated retroperito- neal liposarcoma: a reappraisal of 30 cases formerly classified as well-differentiated liposarcoma. Cancer 43:574-584

9. Murphey MD, Arcara LK, Fanburg-Smith J (2005) From the archives of the AFIP: imaging of musculoskeletal liposarcoma with radiologic-pathologic correlation. Radiographics 25(5):13711395. doi:10.1148/rg.255055106

10. Enzinger FM, Harvey DA (1979) Spindle cell lipoma. Cancer $36: 1852-1859$

11. Kransdorf MJ, Bancroft LW, Peterson JJ, Murphey MD, Foster WC, Temple HT (2002) Imaging of fatty tumors: distinction of lipoma and well-differentiated liposarcoma. Radiology 224(1):99104. doi:10.1148/radiol.2241011113

12. Galant JA, Martí-Bonmatí L, Soler R, Saez F, Lafuente J, Bonmatí C, Gonzalez I (1998) Grading of subcutaneous soft-tissue tumors by means of their relationship with the superficial fascia on MR imaging. Skeletal Radiol 27(12):657-663

13. Ritchie DA, Aniq H, Davies AM, Mangham DC, Helliwell TR (2006) Hibernoma - correlation of histopathology and magneticresonance-imaging features in 10 cases. Skeletal Radiol 35 (8):579-589. doi:10.1007/s00256-006-0114-4

14. Wu JS, Hochman MG (2009) Soft-tissue tumors and tumorlike lesions: a systematic imaging approach. Radiology 253(2):297316. doi:10.1148/radiol.2532081199

15. Gielen JL, De Schepper AM, Parizel PM, Wang XL, Vanhoenacker F (2003) Additional value of magnetic resonance with spin echo T1weighted imaging with fat suppression in characterization of softtissue tumors. J Comput Assist Tomogr 27(3):434-441

16. Galant J, Martí-Bonmatí L, Sáez F, Soler R, Alcalá-Santaella R, Navarro M (2003) The value of fat-suppressed T2 or STIR sequences in distinguishing lipoma from well differentiated liposarcoma. Eur Radiol 13:337-343. doi:10.1007/s00330-0021463-6

17. Chan LP, Gee R, Keogh C, Munk PL (2003) Imaging features of fat necrosis. AJR Am J Roentgenol 181(4):955-959

18. Bancroft LW, Kransdorf MJ, Peterson JJ, Sundaram M, Murphey MD, O'Connor MI (2003) Imaging characteristics of spindle cell lipoma. AJR Am J Roentgenol 181(5):1251-1254 